\title{
Studying corneal epithelium in vivo: a new tool in clinical practice
}

\section{Editorial}

The evaluation of corneal pachymetry has acquired importance in recent years, especially in the preoperative screening of refractive surgery cases. In addition to corneal thickness, studying the distribution of corneal epithelial thickness is becoming a very useful parameter, both clinically and in research settings. Evaluation of the corneal epithelial map can help clinicians in everyday decisionmaking and to a safer screening for refractive surgery candidates. Topographic irregularities of epithelial pachymetry either localized or diffuse, can significantly affect the overall corneal refractive power. In addition, corneal epithelium in several cases may be masking underlying stromal disorders.

Examination tools for studying epithelial thickness comprise several imaging devices such as scanning high frequency ultrasound (HF-UBM, Artemis), confocal microscopy and anterior segment optical coherence tomography (AS-OCT). The later provides optical corneal sections and simultaneously pachymetric data through interpolation of successive radially oriented B-scans. The most advanced AS-OCTs currently use spectral-domain Fourier analysis (SD-OCT), providing significantly higher speed and better resolution compared to earlier, time-domain tomographers (TD-OCT). The additional feature of an AS-OCT system is the 3D imaging of the corneal epithelium. This provides a practical clinical tool for mapping corneal epithelium in vivo, with the advantage of intact, rapid data acquisition. In our center, we use both the RTVue-100 and the Avanti systems (Optovue) as valuable tools for our everyday clinical practice as well as for our clinical research purposes. Our team has published extensive studies on the behavior of the epithelium in a variety of ophthalmic conditions.

\section{The following is a summary of our current knowledge}

The corneal epithelium in normal eyes: The distribution of epithelial maps has been studied in 373 normal subjects. Epithelial pachymetry shows a nearly normal distribution with a mean thickness of 53.28 \pm 3.34 microns (range $45-60 \mu \mathrm{m}$ ), while the topographical variation (variability) is $1.78 \pm 0.78$ microns. The corneal epithelium was also found to be slightly thicker in the lower half of the cornea. A positive correlation between age and epithelial pachymetric abnormality was also observed (increase abnormality of the epithelium with age).

The corneal epithelium in ectasia and keratoconus: It is known from earlier studies with the HF-UBM Artemis system that the corneal epithelium has a compensation function, trying to "cover" topographic anomalies of the underlying stromal layers. The epithelium, for example, becomes thinner over the cone in keratoconic eyes, and correspondingly thicker in the flattest area. This was also identified in our study with AC-OCT. ${ }^{2}$ We have further shown that in keratoconic eyes there is an overall increase in the average corneal epithelial thickness, particularly in younger patients. ${ }^{3}$ Indeed, in our opinion, this "reactive" epithelial hypertrophy can be a very early diagnostic
Volume 4 Issue 2 - 2016

\author{
Constantinos H Karabatsas \\ Professor of Ophthalmology, Laser Vision gr Eye Institute, \\ Greece
}

Correspondence: Constantinos H Karabatsas, Professor of Ophthalmology, LaserVision gr Eye Institute, Athens, Greece, Email karabatsas@hotmail.com

Received: February 01, 2016 | Published: February 03, 2016

marker of subclinical keratoconus before any other topographic (Placido) or tomographic (Scheimpflug) sign becomes evident.

Furthermore, we have shown that the corneal epithelium asymmetry indices and the area of local thinning, both provided by the OCT exam, correlate very well with the anterior corneal keratoconus indices (such as the ISV and IHD indices) provided by Scheimpflug technology. ${ }^{4}$ It is of particular interest our finding that "normalization" of the epithelial topography occurs after a combined excimer laser (customized PRK) and corneal cross-link procedure in keratoconus patients (Athens Protocol). Detailed 12-month postoperative monitoring of keratoconic eyes who underwent Athens protocol, showed an overall thinning and normalization of the epithelial profile (approaching absolutely normal values) compared to untreated keratoconic eyes (Figure 1). ${ }^{5}$

The corneal epithelium in dry eye: We have observed an overall increase in epithelial thickness in dry eyes (average $58 \mu \mathrm{m}$ ). The topographic variance is comparable to the normal eye (mean of $3 \mu \mathrm{m}$ ). These findings are likely to play a key role in the preoperative and postoperative assessment of refractive patients and in some cases may precede the appearance of other specific dry eye-tests such as TBUT and Schirmer's. ${ }^{6}$

The corneal epithelium following cataract surgery: We have analyzed corneal epithelium with AS-OCT, preoperatively and 3 months postoperatively, in 116 eyes undergoing cataract surgery. Due to epithelial remodeling occuring after cataract surgery, the epithelium returns to preoperative values at the third postoperative month. Delayed remodeling was seen in elderly (age $>75$ years) patients. ${ }^{7}$

The corneal epithelium after LASIK: Changes in the thickness of the corneal epithelium following myopic LASIK correction with mechanical microkeratome have been reported in previous studies. The increase in the central epithelium thickness observed after LASIK, was attributed to refractive regression. In our own studies we have seen only a mild increase (average $3 \mu \mathrm{m}$ ) of the epithelium thickness, which was rather non-lenticular, and more evident in the middle corneal region, at $5 \mathrm{~mm}$ diameter area. Moreover, a positive correlation with the amount of attempted correction was found; the higher the attempted myopic correction and therefore the flattening of the 
cornea, the greater the increase in epithelial thickness. ${ }^{8}$ Comparison of the postoperative epithelium between patients undertaking 'standard' LASIK vs. LASIK with simultaneous prophylactic corneal crosslink (Lasik-Xtra), revealed that when cornea cross-linking is applied, no corresponding increase in the epithelial thickness is observed. ${ }^{9}$ The differences found, cannot be fully explained according to the theory of epithelial compensation response. One explanation which we propose might be the reduced epithelial hyperactivity as a result of increased structural bio-tectonics (rigidity) of corneas following cross linking. This may not allow large oscillations in the cornea.

Certainly, much remains to be studied and explained in relation to the behavior of the epithelium in various corneal pathologies. However, technological advancements such as the AC-OCT, provide us with the ability of in vivo detailed information.

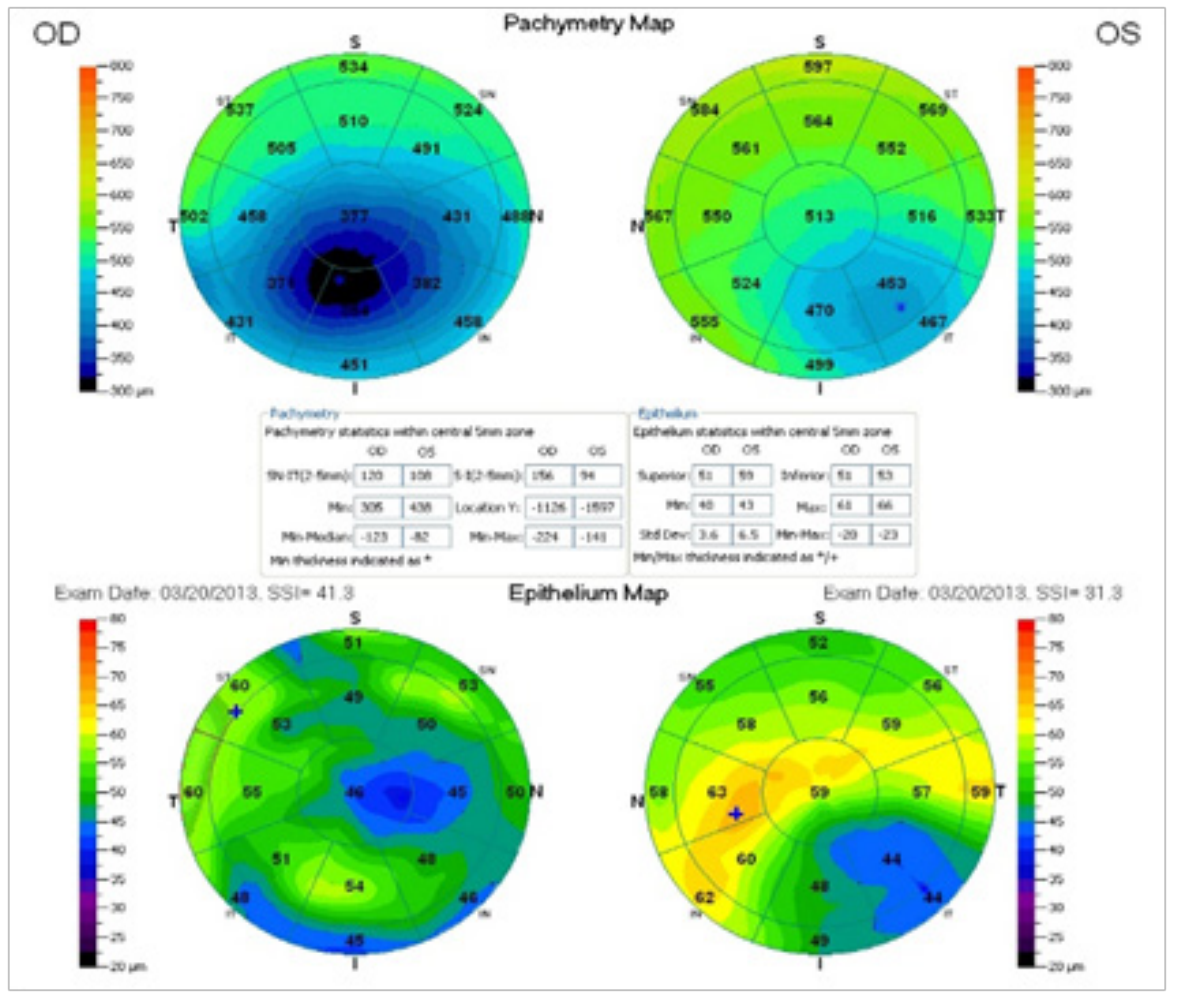

Figure I OCT pachymetric (upper) and epithelial (lower) maps of the same patient suffering from keratoconus. The right eye (OD) has undertaken Athens protocol procedure, while the left eye (OS) not.

\section{Acknowledgments}

None.

\section{Conflicts of interest}

No conflicts to declare.

\section{Funding}

None.

\section{References}

1. Kanellopoulos AJ, Asimellis G. In vivo three-dimensional epithelial imaging of corneal epithelium in normal eyes by anterior segment optical coherence tomography: a clinical reference study. Cornea. 2013;32(11):1493-1498.

2. Kanellopoulos AJ, Asimellis G. Anterior segment optical coherence tomography - assisted topographic corneal epithelial thickness distribution imaging of a keratoconus patient. Case Rep Ophthalmol. 2013;4(1):74-78.

3. Kanellopoulos AJ, Aslanides IM, Asimellis G. Correlation between epithelial thickness in normal corneas, ectatic and ectatic previously treated with CXL corneas. Can overall epithelial thickness become a very early ectasia prognostic factor? Clin Ophthalmol. 2012;6:789-800.
4. Kanellopoulos AJ, Asimellis G. OCT-derived Comparison of Corneal Thickness Distribution and Asymmetry Differences between Normal and Keratoconic Eyes. Cornea. 2014;33(12):1274-1281.

5. Kanellopoulos AJ, Asimellis G. Epithelial remodeling after partial topography-guided normalization and high-fluence short-duration crosslinking (Athens protocol): Results up to 1 year. $J$ Cataract Refract Surg. 2014;40(10):1597-1602.

6. Kanellopoulos AJ, Asimellis G. In Vivo 3-Dimensional Corneal Epithelial Thickness Mapping as an Indicator of Dry Eye: Preliminary Clinical Assessment. Am J Ophthalmol. 2014;157(1):63-68.

7. Kanellopoulos AJ, Asimellis G. Corneal Epithelial Remodeling Following Cataract Surgery: Three-Dimensional Investigation with Anterior-Segment Optical Coherence Tomography. J Refract Surg. 2014;30(5):348-353.

8. Kanellopoulos AJ, Asimellis G. Longitudinal postoperative lasik epithelial thickness profile changes in correlation with degree of myopia correction. J Refract Surg. 2014;30(3):166-171.

9. Kanellopoulos AJ, Asimellis G. Epithelial Remodeling After Femtosecond Laser-assisted High Myopic LASIK: Comparison of Stand-alone with LASIK Combined with Prophylactic High-fluence Cross-linking. Cornea. 2014;33(5):463-469. 